10,12

\title{
Изменение термодинамических свойств при изохорическом и изобарическом уменьшении размера нанокристалла кремния
}

\section{(C) М.Н. Магомедов}

Институт проблем геотермии ДагНЦ РАН, Махачкала, Россия

E-mail: mahmag4@mail.ru

(Поступила в Редакцию 25 октября 2018 г. В окончательной редакции 29 ноября 2018 г. Принята к публикации 29 ноября 2018 г.)

На основе метода расчета свойств кристалла из парного межатомного потенциала Ми-Леннард-Джонса и RP-модели нанокристалла рассчитано уравнение состояния $P\left(v / v_{o}\right)$ и барические зависимости как решеточных, так и поверхностных свойств для макро- и для нанокристаллов кремния. Показано, что при определенном значении относительного объема $\left(v / v_{o}\right)_{0}$ изотермические зависимости $P\left(v / v_{o}\right)$ для макрои нанокристалла пересекаются. В точке пересечения (при $\left.\left(v / v_{o}\right)_{0}\right)$ поверхностное давление становится равным нулю. Величина $\left(v / v_{o}\right)_{0}$ уменьшается как при изоморфно-изомерном росте температуры, так и при изоморфно-изотермическом уменьшении $N-$ числа атомов в нанокристалле, или при изомерноизотермическом отклонении формы нанокристалла от наиболее энергетически оптимальной формы (для $\mathrm{RP}$-модели это куб). Исходя из полученного уравнения состояния, изучено изменение свойств кремния как при изохорическом $\left(v / v_{o}=1\right)$, так и при изобарическом $(P=0)$ уменьшении $N$ при температуpax 300 и $1000 \mathrm{~K}$.

Работа выполнена при финансовой поддержке РФФИ в рамках научного проекта № 18-29-11013_мк и Программы Президиума РАН (программа № I.13).

DOI: $10.21883 /$ FTT.2019.04.47426.267

\section{1. Введение}

Известно, что при уменьшении размера нанокристалла его свойства изменяются [1-3]. При этом наименее изучены размерные зависимости барических свойств. Поэтому в данной работе мы изучим изменение с размером, как уравнения состояния, так и барических зависимостей решеточных и поверхностных свойств нанокристалла кремния.

Экспериментальное изучение размерных зависимостей проводят, как правило, в изобарических условиях [1], т.е. когда давление в системе постоянно: $P=$ const. Вместе с тем теоретические расчеты проводят, в большинстве работ, для изохорического процесса (т.е. при постоянной плотности: $R=\mathrm{const}$ ) [1-3]. Это связано с тем, что в эксперименте трудно сохранить постоянной плотность нанокристалла, а в теоретических моделях трудно было определить поверхностное давление, а потому и уравнение состояния нанокристалла. В связи с этим, возникли следующие вопросы.

1. Насколько велика разница в изменении того или иного свойства при изохорическом и при изобарическом уменьшении размера нанокристалла.

2. Какие свойства изменяются слабо при изохорическом либо изобарическом уменьшении размера нанокристалла.
Поэтому в данной работе мы изучим размерное изменение различных свойств кремния, как при изохорическом, так и при изобарическом уменьшении числа атомов в нанокристалле при различных температурах.

\section{2. Метод расчета решеточных свойств нанокристалла}

Для расчета решеточных свойств нанокристалла простого однокомпонентного вещества необходимо определить, как потенциал взаимодействия пары его атомов, так и метод расчета, который основан на этом потенциале. Представим парное межатомное взаимодействие в кристалле однокомпонентного вещества в виде потенциала Ми-Леннард-Джонса, который имеет следующий вид $[4$, гл. 3]:

$$
\varphi(r)=\frac{D}{(b-a)}\left[a\left(\frac{r_{o}}{r}\right)^{b}-b\left(\frac{r_{o}}{r}\right)^{2}\right],
$$

где $D$ и $r_{o}$ - глубина и координата минимума потенциала, $b>a>1$ - параметры.

Тогда, используя приближение „взаимодействия только ближайших соседей“ температуру Дебая как функцию первого координационного числа и расстояния между центрами ближайших атомов можно определить из вы- 
ражения $[4$, гл. 2;5]:

$$
\Theta\left(k_{B}, c\right)=A_{w}\left(k_{n}, c\right) \xi\left[-1+\left(1+\frac{8 D}{k_{B} A_{w}\left(k_{n}, c\right) \xi^{2}}\right)^{1 / 2}\right],
$$

где $k_{B}$ - постоянная Больцмана, функция $A_{w}$ возникает из-за учета энергии „нулевых колебаний“ атомов в кристалле, $k_{n}$ - первое координационное число, которое в нанокристалле зависит как от его размера, так и от формы его поверхности $\left[4\right.$, гл. 6], $c=\left(6 k_{p} v / \pi\right)^{1 / 3}-$ расстояние между центрами ближайших атомов, $v=V / N-$ удельный объем, $V$ и $N$ - объем и число атомов в нанокристалле

$$
\begin{gathered}
A_{w}\left(k_{n}, c\right)=K_{R} \frac{5 k_{n} a b(b+1)}{144(b-a)}\left(\frac{r_{o}}{c}\right)^{b+2}, \\
K_{R}=\frac{\hbar^{2}}{k_{B} r_{o}^{2} m}, \quad \xi=\frac{9}{k_{n}(\infty)},
\end{gathered}
$$

$m$ - масса атома, $\hbar-$ постоянная Планка, $k_{p}-$ коэффициент упаковки структуры, $k_{n}(\infty)-$ первое координационное число в объеме нанокристалла.

Если для нанокристалла с геометрической поверхностью Гиббса применимо приближение „взаимодействия только ближайших соседей“, то зависимость энергии нанокристалла от размера и формы будет определяться зависимостью от размера и формы среднего (по нанокристаллу) значения первого координационного числа. В этом случае, используя для колебательного спектра нанокристалла модель Эйнштейна, для удельной (на атом) свободной энергии Гельмгольца нанокристалла можно принять выражение $[4,6]$

$$
\begin{aligned}
& f_{H}\left(k_{n}, c, T\right)=\left(\frac{k_{n}}{2}\right) D U(R)+3 k_{B} \Theta_{E}\left(k_{n}, c\right) \\
& \quad \times\left\{\left(\frac{1}{2}\right)+\left(\frac{T}{\Theta_{E}\left(k_{n}, c\right)}\right) \ln \left[1-\exp \left(-\frac{\Theta_{E}\left(k_{n}, c\right)}{T}\right)\right]\right\},
\end{aligned}
$$

где $\Theta_{E}$ - это температура Эйнштейна, которая связана с температурой Дебая соотношением [6]: $\Theta=(4 / 3) \Theta_{E}$, $R=r_{o} / c-$ относительная линейная плотность кристалла, функция потенциальной энергии, в соответствии с (1), равна

$$
U(R)=\frac{a R^{b}-b R^{a}}{b-a} .
$$

Положим, что зависимость функции $k_{n}$ от размера и формы нанокристалла с поверхностью Гиббса не зависит от плотности и температуры, а определяется только числом атомов в нанокристалле $(N)$, параметром его формы $(f)$ и постоянными $k_{n}(\infty)$ и $k_{p}$. Тогда из $(4)$ для термического уравнения состояния $(P)$ и изотермического модуля упругости $\left(B_{T}\right)$ можно получить [7]:

$$
P=-\left(\frac{\partial f_{H}}{\partial v}\right)_{T}=\left[\frac{k_{n}}{6} D U^{\prime}(R)+3 k_{B} \Theta_{E} \gamma E_{w}\left(\frac{\Theta_{E}}{T}\right)\right] \frac{1}{v},
$$

$$
\begin{aligned}
B_{T}= & -v\left(\frac{\partial P}{\partial v}\right)_{T} \\
= & P+\left[\frac{k_{n}}{18} D U^{\prime \prime}(R)+3 k_{B} \Theta_{E} \gamma(\gamma-q) E_{w}\left(\frac{\Theta_{E}}{T}\right)\right. \\
& \left.-3 N k_{B} \gamma^{2} T F_{E}\left(\frac{\Theta_{E}}{T}\right)\right] \frac{1}{v} .
\end{aligned}
$$

Здесь введены следующие функции

$$
\begin{gathered}
F_{E}(y)=\frac{y^{2} \exp (v)}{[\exp (y)-1]^{2}}, E_{w}(y)=0.5+\frac{1}{[\exp (y)-1]}, \\
U^{\prime}(R)=R\left[\frac{\partial U(R)}{\partial R}\right]=\frac{a b\left(R^{b}-R^{a}\right)}{b-a}, \\
U^{\prime \prime}(R)=R\left[\frac{\partial U^{\prime}(R)}{\partial R}\right]=\frac{a b\left(b R^{b}-a R^{a}\right)}{b-a} .
\end{gathered}
$$

Из (2) легко найти выражения для первого $(\gamma)$, второго $(q)$ и третьего $(z)$ параметров Грюнайзена

$$
\begin{gathered}
\gamma=-\left(\frac{\partial \ln \Theta}{\partial \ln v}\right)_{T}=\frac{b+2}{6\left(1+X_{w}\right)}, \\
q=\left(\frac{\partial \ln \gamma}{\partial \ln v}\right)_{T}=\gamma \frac{X_{w}\left(1+2 X_{w}\right)}{\left(1+X_{w}\right)}, \\
z=-\left(\frac{\partial \ln q}{\partial \ln v}\right)_{T}=\gamma\left(1+4 X_{w}\right)-2 q \\
=\gamma\left(\frac{1+3 X_{w}}{1+X_{w}}\right)=\frac{(b+2)}{6} \frac{\left(1+3 X_{w}\right)}{\left(1+X_{w}\right)^{2}},
\end{gathered}
$$

где введена функция: $X_{w}=A_{w} \xi / \Theta$, которая определяет роль квантовых эффектов.

Так как температура Дебая (2) не зависит от температуры при изохорическом нагреве, то изохорную и изобарную теплоемкости можно определить в виде [6]:

$$
C_{v}=3 N k_{B} F_{E}\left(\frac{\Theta_{E}}{T}\right), C_{p}=C_{v}\left(1+\gamma \alpha_{p} T\right),
$$

где изобарный коэффициент теплового объемного расширения можно рассчитать по уравнению Грюнайзена [6]:

$$
\alpha_{p}=\left(\frac{\gamma}{k_{a}}\right) \frac{C_{v}}{V B_{T}}=\frac{\gamma C_{v}}{k_{a} N B_{T}\left[\pi r_{o}^{3} /\left(6 k_{p}\right)\right]}\left(\frac{v_{o}}{v}\right), v_{o}=\frac{\pi r_{o}^{3}}{6 k_{p}} .
$$

Здесь введен корректирующий множитель: $k_{a}=\gamma / \gamma_{C}$. Он возникает из-за того, что „частотный“ параметр Грюнайзена (который рассчитывается из температуры Дебая по формуле (8)) может отклоняться от термодинамического параметра Грюнайзена $\gamma_{C}$ (который рассчитывается по термодинамическим данным, входящим в (10)) $[8,9]$.

Полученные выражения (2)-(10) позволяют рассчитать зависимость, как уравнения состояния, так и указанных решеточных свойств от нормированного объема 
$v / v_{o}=\left(c / r_{o}\right)^{3}=R^{-3}$ и температуры $T$ для однокомпонентного нанокристалла с данной структурой (т.е. при данных значениях $k_{n}(\infty)$ и $k_{p}$ ) если известны четыре параметра межатомного потенциала (1) и функциональная зависимость первого координационного числа от размера и формы нанокристалла, т. е. функция $k_{n}(N, f)$.

Отметим, что если принять: $N \rightarrow \infty, V \rightarrow \infty$ при $v=V / N=$ const, и $k_{n}(N, f)=k_{n}(\infty)$, то формализм из (2)-(10) позволяет рассчитать как уравнение состояния, так и барические зависимости термодинамических свойств макрокристалла. Этот метод был успешно применен при расчете свойств макрокристаллов железа, алмаза, кремния, германия [7] и молибдена [10].

\section{RР-модель нанокристалла}

Для определения функции $k_{n}(N, f)$ используем $\mathrm{RP}$-модель нанокристалла $[4,11]$, суть которой в следующем. Положим, что нанокристалл со свободной поверхностью Гиббса имеет вид прямоугольного параллелепипеда с квадратным основанием, ограненный гранями типа (100). Величина $f=N_{p s} / N_{p o}-$ это параметр формы, который определяется отношением числа атомов на боковом ребре $N_{p s}$ к числу атомов на ребре основания $N_{p o}$. Для стержневидной формы $f>1$, для куба $f=1$, для нанокристалла пластинчатой формы $f<1$. Число атомов в нанокристалле, равное: $N=f N_{p o}^{3} / \alpha$, изменяется в пределах: $2^{3} / \alpha \leq N \leq \infty$, где $\alpha=\pi /\left(6 k_{p}\right)$ - параметр структуры.

Ограничение системы поверхностью приведет к обрыву связей на границе. Поэтому если использовано приближение взаимодействия „только ближайших соседей ${ }^{\prime}$, то вместо первого координационного числа $k_{n}(\infty)$ необходимо брать $k_{n}(N, f)$ - среднее (по нанокристаллу) значение первого координационного числа, которое будет зависеть как от $N$, так и от $f[4,11]$. При этом структуру системы (характеризующуюся коэффициентом упаковки $k_{p}$ ) полагаем неизменной: $k_{p}=$ const.

B рамках RP-модели зависимость нормированного среднего значения первого координационного числа от размера $(N)$ и формы $(f)$ нанокристалла имеет вид $[4,11]$ :

$$
k_{n}^{*}=\frac{k_{n}(N, f)}{k_{n}(\infty)}=1-Z_{s}(f)\left(\frac{\alpha^{2}}{N}\right)^{1 / 3},
$$

где $k_{n}(\infty)=k_{n}(N=\infty)$ - координационное число для макрокристалла,

$$
Z_{s}(f)=\frac{1+2 f}{3 f^{2 / 3}}
$$

Функция формы $Z_{s}(f)$ достигает минимума равного единице при $f=1$, т. е. при форме куба. Для пластинчатых $(f<1)$ или стержневидных $(f>1)$ форм значение $Z_{s}(f)$ больше единицы. Поэтому функция $k_{n}(f)^{*}$ из (11) при любом значении $N$ имеет максимум при $f=1$, т. е. для наиболее энергетически оптимальной кубической формы прямоугольного параллелепипеда.

Объем и площадь поверхности для RP-модели равны

$$
V=N_{p o}^{3} f c^{3}=N \alpha c^{3}, \quad \Sigma=6 c^{2} \alpha_{s}(N \alpha)^{2 / 3} Z_{s}(f),
$$

где $\alpha_{s}-$ коэффициент, учитывающий плотность упаковки атомов на грани (т.е. в поверхностном слое) нанокристалла: $\alpha_{s} \cong \alpha^{2 / 3}$. Легко видеть, что объем нанокристалла $V$ не зависит от формы системы, т.е. от величины $f$.

В рамках RР-модели для $\sigma(N, f)$ - удельной (на единицу площади) поверхностной энергии грани (100) нанокристалла, ее изохорной и изобарной производных по температуре, и для поверхностного давления $\left(P_{s f}\right)$ были получены выражения следующего вида $[4$, гл. $6 ; 11]$ :

$$
\begin{gathered}
\sigma(N, f)=-\frac{k_{n}(\infty) D R^{2}}{12 \alpha^{2 / 3} r_{o}^{2}} L_{E}(N, f), \\
\sigma^{\prime}(T)_{v}=\left(\frac{\partial \sigma}{\partial T}\right)_{c, N, f} \\
=-\frac{3 k_{B} R^{2} \gamma(N, f)}{2 \alpha^{2 / 3}(b+2) r_{o}^{2} k_{n}(N, f)^{*}} F_{E}\left(\frac{\Theta_{E}}{T}\right), \\
\sigma^{\prime}(T)_{P}=\left(\frac{\partial \sigma}{\partial T}\right)_{P, N, f}=\sigma^{\prime}(T)_{v}+v \alpha_{p}\left(\frac{\partial \sigma}{\partial v}\right)_{T, N, f} \\
=\sigma^{\prime}(T)_{v}-\frac{2}{3} \sigma \alpha_{p} \Delta_{p}, \\
P_{s f}=\left[\frac{\partial(\sigma \Sigma / N)}{\partial v}\right]_{T, N}=P_{l s}\left(1-\Delta_{p}\right),
\end{gathered}
$$

где давление Лапласа $P_{l s}$ и введенные функции имеют вид

$$
\begin{gathered}
P_{l s}=\frac{2 \Sigma}{3 V} \sigma=\frac{4 \alpha_{s} Z_{s}(f)}{(\alpha N)^{1 / 3} c} \sigma=4 \alpha_{s} \frac{\left(1-k_{n}^{*}\right)}{\alpha c} \sigma, \\
L_{E}(N, f)=U(R)+3 H_{w}(N, T), \\
\Delta_{p}=-\frac{1}{2}\left[\frac{\partial \ln (\sigma)}{\partial \ln (c)}\right]_{T, N, k_{p}, f}=1+\frac{1}{2 L_{E}(N, f)} \\
\times\left\{U^{\prime}(R)-9\left[q-\gamma t_{y}\left(\frac{\Theta_{E}}{T}\right)\right] H_{w}(N, T)\right\}, \\
H_{w}(N, T)=\frac{6 \gamma(N, f)}{(b+2)}\left[\frac{k_{B} \Theta_{E}(N, f)}{D k_{B}(N, f)}\right] E_{w}\left(\frac{\Theta_{E}}{T}\right), \\
t_{y}(y)=1-\frac{2 y \exp (y)}{[\exp (2 y)-1]} .
\end{gathered}
$$

При положительном значении $P_{s f}$ поверхностное давление сжимает нанокристалл, как это обычно и допускается. Очевидно, что в „термодинамическом пределе“ (т. е. когда $N \rightarrow \infty$ и $V \rightarrow \infty$ при $v=\mathrm{const)} \mathrm{функции} P_{l s}$ из (17) и $P_{s f}$ из (16) исчезают, ибо в этом случае: $k_{n}^{*}(N \rightarrow \infty) \rightarrow 1$, а выражения из (13)-(15) переходят в формулы для макрокристалла. При $T \rightarrow 0 \mathrm{~K}$ функции 
из (14) и (15) стремятся к нулю при любом $N$, что согласуется с третьим началом термодинамики в „сильной“ формулировке Планка.

Отметим, что поверхностное давление также можно найти и из выражения (5), как разницу между давлениями, рассчитанными для макро- и нанокристалла:

$$
P_{s f}=P(T, v, N=\infty)-P(T, v, N, f) .
$$

Таким образом, полученная в рамках RP-модели функция $k_{n}(N, f)$ вместе с формализмом из (2)-(20) позволяют рассчитать зависимость всех решеточных и поверхностных свойств от размера и формы нанокристалла при данных значениях температуры и удельного объема.

\section{4. Результаты расчета свойств кремния}

Для расчетов был выбран кремний $(m(\mathrm{Si})=$ $=28.09$ a.m.u.), имеющий структуру алмаза: $k_{n}(\infty)=4$, $k_{p}=0.3401$. Параметры парного межатомного потенциала (1) были определены в [4, гл. 3; 12] и апробированы в работах [7,11,12]: $r_{o}=0.2351 \mathrm{~nm}, b=4, a=2.48$, $D_{b} / k_{B}=64286.16 \mathrm{~K}, D_{s} / k_{B}=26921.28 \mathrm{~K}$. Здесь $D_{b}$ глубина потенциала, который работает при упругой (обратимой) деформации ковалентного кристалла, а $D_{s}-$ глубина потенциала для расчета свойств кристалла при его пластической (необратимой) деформации.

На рисунке показаны рассчитанные с помощью потенциала упругого типа (т.е. с глубиной $D_{b}$ ) и выражения (5) две изотермические зависимости давления от нормированного объема $\left(v / v_{o}\right)$ для кремния. Две нижние линии 1 и $2-$ изотермы $T=300 \mathrm{~K}$, две верхние линии 3 и 4 - изотермы $T=1000 \mathrm{~K}$. На рисунке сплошные линии 1 и 3 получены для макрокристалла,

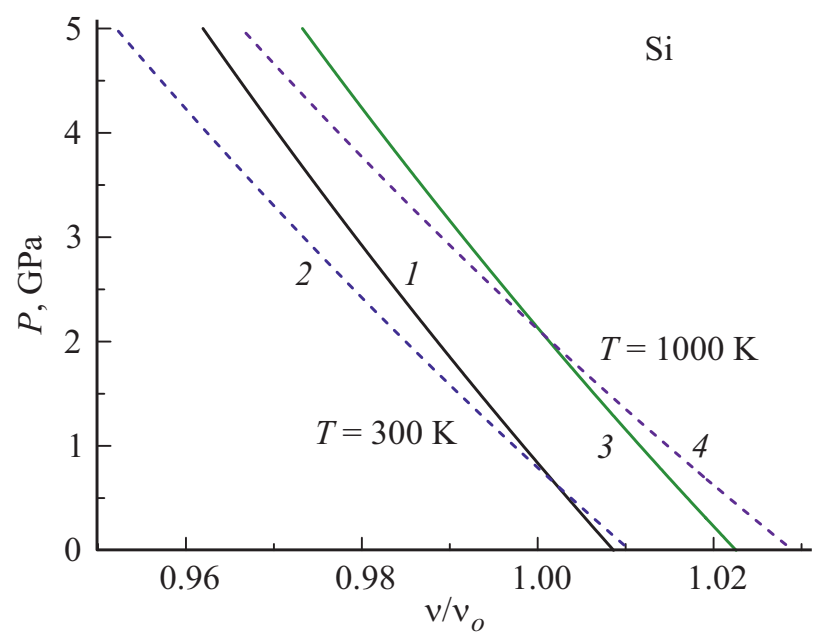

Изотермические зависимости давления от относительного объема для макро- (линии 1 и 3 ) и нанокристалла (линии 2 и 4 ) кремния, полученные на основе потенциала упругого типа $\left(\right.$ при $\left.D_{b}\right)$. Две нижние линии 1 и $2-$ изотермы $T=300 \mathrm{~K}$, две верхние линии 3 и $4-$ изотермы $T=1000 \mathrm{~K}$.
Таблица 1. Координаты точки нулевого поверхностного давления, где $P_{s f}=P($ Macro $)-P(N, f)=0$, полученные для кубического нанокристалла кремния из $N=223$ атомов $\left(N_{p o}=7\right.$, $f=1)$ на основе потенциала упругого типа (при $D_{b}$ )

\begin{tabular}{c|c|c|c|c}
\hline$T, \mathrm{~K}$ & $R_{0}=r_{o} / c_{0}$ & $\left(v / v_{o}\right)_{0}$ & $v\left(P_{s f}=0\right), \mathrm{cm}^{3} / \mathrm{mol}$ & $P_{0}, \mathrm{GPa}$ \\
\hline 300 & 0.9993 & 1.0021 & 12.073 & 0.6289 \\
1000 & 0.9998 & 1.0007 & 12.056 & 2.0622
\end{tabular}

а пунктирные линии 2 и 4 - изоморфно-изомерные (т.е. при постоянных значениях $f$ и $N$ ) зависимости для нанокристалла из $N=223$ атомов с кубической формой поверхности Гиббса, т. е. при $N_{p o}=7$ и $f=1$.

Сравнение рассчитанной таким путем зависимости $P\left(v / v_{o}, T, N=\infty\right)$ с экспериментальными данными для макрокристалла кремния было проведено нами в [7], поэтому здесь мы этого делать не будем. Уменьшение роста давления при переходе от макро- к нанокристаллу указывает на уменьшение модуля упругости: $B_{T}=-v(\partial P / \partial v)_{T}$, с уменьшением размера. На уменьшение $B_{T}$ было указано также и другими методами в работах [2,11,13-16]. Отметим, что поверхностное давление, рассчитанное по формуле (16) с точностью до третьего знака совпало с результатом, полученным из выражения (21). Это также подтверждает корректность данного метода расчета уравнения состояния нанокристалла.

Из рисунка видно, что при определенном значении относительного объема $\left(v / v_{o}\right)_{0}$ изотермические зависимости $P\left(v / v_{o}\right)$ для нанокристалла и макрокристалла пересекаются. Таким образом, при $\left(v / v_{o}\right)_{0}$ поверхностное давление становится равным нулю: $P_{s f}\left(v / v_{o}\right)_{0}=P($ Macro $)-P(N=223, f=1)=0$ следовательно: $\Delta_{p}\left(v / v_{o}\right)_{0}=1$. При $v / v_{o}<\left(v / v_{o}\right)_{0}$ поверхностное давление сжимает нанокристалл $\left(P_{s f}>0\right)$, а при $v / v_{o}>\left(v / v_{o}\right)_{0}$ поверхностное давление нанокристалл растягивает: $P_{s f}<0$.

В табл. 1 представлены координаты точки „нулевого поверхностного давления“, полученные с потенциалом упругого типа (т.е. при $D_{b}$ ) для кубического нанокристалла кремния из $N=223$ атомов $\left(N_{p o}=7, f=1\right)$. Расчеты показали, что величина $\left(v / v_{o}\right)_{0}$ уменьшается как при изоморфно-изомерном ( $f, N$ - const) poсте температуры, так и при изоморфно-изотермическом $(f, T$ - const $)$ уменьшении $N$, или при изомерноизотермическом ( $N, T$ - const) отклонении формы нанокристалла от наиболее энергетически оптимальной формы (для RP-модели это куб).

Из рисунка также следует, что давление в нанокристалле переходит через ноль при значении $v / v_{o}$ бо́льшем, чем для макрокристалла, т.е. при изобарическом уменьшении числа атомов нанокристалл становится более растянутым отрицательным поверхностным давлением, чем макрокристалл. Поэтому полагать, что при изобарическом уменьшении числа атомов плотность 
Таблица 2. Рассчитанные при $T=300 \mathrm{~K}$ значения свойств (указаны в первом столбце) для Si. Расчеты сделаны на основе потенциала упругого типа (при $D_{b}$ ). Во втором и третьем столбцах показаны абсолютные результаты для макрокристалла для изохоры $R=1$ и для изобары $P=0$ соответственно. В четвертом и пятом столбцах - относительные (к соответствующим значениям для макрокристалла) значения свойств для кубического нанокристалла из $N=223$ атомов $\left(\right.$ при $\left.N_{p o}=7, f=1\right)$

\begin{tabular}{|c|c|c|c|c|}
\hline Размер $=$ & \multicolumn{2}{|c|}{ Macro $N=\infty$} & \multicolumn{2}{|c|}{ Nano $N=223\left(N_{p o}=7, f=1\right)$} \\
\hline $\begin{array}{c}\text { Свойство } \\
\text { [единица измерения] }\end{array}$ & $\begin{array}{c}R=r_{o} / c=1 \\
P=0.836 \mathrm{GPa}\end{array}$ & $\begin{array}{c}P=0 \\
R=0.9972\end{array}$ & $\begin{array}{c}X(N, R=1) / X(\infty) \\
P(N)=0.791 \mathrm{GPa} \\
P_{s f}=0.045 \mathrm{GPa}\end{array}$ & $\begin{array}{c}X(N, P(N)=0) / X(\infty) \\
R=0.9965 \\
P_{s f}=-0.175 \mathrm{GPa}\end{array}$ \\
\hline $\begin{array}{l}v\left[\mathrm{~cm}^{3} / \mathrm{mol}\right] \\
\Theta[\mathrm{K}] \\
\gamma \\
q \cdot 10^{3} \\
z \\
B_{T}[\mathrm{GPa}] \\
B^{\prime}(P)^{1)} \\
\alpha_{p}\left[10^{-6} \mathrm{~K}^{-1}\right] \\
\alpha_{p} B_{T}\left[10^{-3} \mathrm{GPa} / \mathrm{K}\right] \\
C_{v} /\left(N k_{B}\right) \\
C_{p} /\left(N k_{B}\right) \\
\Theta^{\prime}(P)[\mathrm{K} / \mathrm{GPa}]^{1)} \\
C_{v}^{\prime}(P) /\left(N k_{B}\right)\left[10^{-3} / \mathrm{GPa}\right]^{1)} \\
\alpha_{p}^{\prime}(P)\left[10^{-8} /(\mathrm{K} \cdot \mathrm{GPa})\right]^{1)} \\
C_{p}^{\prime}(P) /\left(N k_{B}\right)\left[10^{-3} / \mathrm{GPa}\right]^{1)} \\
\sigma(100)\left[10^{-3} \mathrm{~J} / \mathrm{m}^{2}\right]^{2)} \\
\sigma^{\prime}(T)_{v}\left[10^{-6} \mathrm{~J} /\left(\mathrm{m}^{2} \mathrm{~K}\right)\right]^{2)} \\
\sigma^{\prime}(T)_{P}\left[10^{-6} \mathrm{~J} /\left(\mathrm{m}^{2} \mathrm{~K}\right)\right]^{2)} \\
\sigma^{\prime}(P)_{T}\left[10^{-3} \mathrm{~J} /\left(\mathrm{m}^{2} \mathrm{GPa}\right)\right]^{1 / 2)} \\
\sigma /\left(c \cdot B_{T}\right)^{2)} \\
\mu_{p}^{2)}\end{array}$ & $\begin{array}{r}12.0476 \\
850.185 \\
0.99627 \\
3.74736 \\
1.00371 \\
99.0400 \\
4.13139 \\
7.46572 \\
0.73941 \\
2.08628 \\
2.09093 \\
8.55191 \\
-14.7053 \\
-28.8883 \\
-14.9185 \\
1665.18 \\
-39.8972 \\
-63.9262 \\
26.4255 \\
0.16926 \\
0.37548\end{array}$ & $\begin{array}{r}12.1515 \\
842.942 \\
0.99630 \\
3.71520 \\
1.00367 \\
95.5728 \\
4.16755 \\
7.71647 \\
0.73749 \\
2.09874 \\
2.10358 \\
8.78507 \\
-15.1073 \\
-31.1470 \\
-15.3377 \\
1655.64 \\
-39.7739 \\
-64.7312 \\
27.6113 \\
0.17386 \\
0.37879\end{array}$ & $\begin{array}{l}1.0000 \\
0.8836 \\
1.000438 \\
0.8828 \\
0.9996 \\
0.7816 \\
0.9987 \\
1.3832 \\
1.0810 \\
1.0806 \\
1.0815 \\
1.1308 \\
1.0973 \\
1.7005 \\
1.1072 \\
0.9976 \\
1.3264 \\
1.3231 \\
1.2734 \\
1.2735 \\
1.0716\end{array}$ & $\begin{array}{l}1.0018 \\
0.8820 \\
1.000440 \\
0.8812 \\
0.9996 \\
0.7758 \\
1.0005 \\
1.3906 \\
1.0788 \\
1.0803 \\
1.0813 \\
1.1369 \\
1.0997 \\
1.7260 \\
1.1105 \\
0.9964 \\
1.3247 \\
1.3256 \\
1.2850 \\
1.2805 \\
1.0704\end{array}$ \\
\hline
\end{tabular}

Примечание. ${ }^{1)}$ Рассчитано путем численного дифференцирования данного параметра по давлению вдоль изотермы. ${ }^{2)}$ Данные параметры рассчитаны при указанных значениях $R$, но при использовании межатомного потенциала пластичного вида, т. е. при $D_{s}$.

нанокристалла сохраняется некорректно. При этом, чем дальше от точки „нулевого поверхностного давления“ лежит значение давления изобары, тем больше будет меняться плотность нанокристалла при изотермоизоморфном уменьшении в нем числа атомов.

В табл. 2 и 3 представлены рассчитанные по вышеописанной методике значения решеточных и поверхностных свойств (указаны в первом столбце) для кремния при $T=300$ и $T=1000 \mathrm{~K}$. Во втором и третьем столбцах показаны абсолютные значения свойств макрокристалла для изохоры $v / v_{o}=1$ и для изобары $P=0$ соответственно. В четвертом и пятом столбцах - относительные (к соответствующим значениям для макрокристалла) значения свойств для нанокристалла кубической формы из $N=223$ атомов (т.е. при $N_{p o}=7$, $f=1)$, как для изохоры $v / v_{o}=1$, так и для изобары $P(N)=0$.

Корректирующий множитель $k_{a}$ был определен путем сопоставления расчетного $\alpha_{p}$ (calc) и экспериментального $\alpha_{p}(\exp )$ значений коэффициента теплового расширения макрокристалла кремния при $T=300 \mathrm{~K}$ и $P=0$ : $k_{a}=\alpha_{p}(\mathrm{calc}) / \alpha_{p}(\exp )$. Используя данные из работы [17]: $\alpha_{p}(\exp )=7.6683 \cdot 10^{-6} 1 / \mathrm{K}$, было получено: $k_{a}=1.94$. При расчетах предполагалось, что величина $k_{a}$ не зависит от температуры, давления и числа атомов в нанокристалле.

Расчеты поверхностной энергии и ее производных по $T$ были проведены на основе потенциала пластичного типа, т.е. с глубиной $D_{s}$. Это обусловлено тем, что, как на это было указано в $[4,7,11,12]$, при измерении поверхностной энергии кристалла происходит необратимый разрыв ковалентных связей.

Производные параметра по давлению были рассчитаны путем численного дифференцирования данного параметра по давлению вдоль изотермы.

Зависимость коэффициента Пуассона от размера нанокристалла при различных температурах оценивалась из соотношения, полученного в работе [18]:

$$
\mu_{p}(N, T)=\frac{1}{2}-\frac{1}{48 X_{c}(N, T)[\gamma(N)]^{2}},
$$

где введено обозначение:

$$
X_{c}(N, T)=\frac{\sigma(N, T)}{c B_{T}(N, T)}
$$


Таблица 3. Рассчитанные при $T=1000 \mathrm{~K}$ значения свойств (указаны в первом столбце) для Si. Расчеты сделаны на основе потенциала упругого типа (при $D_{b}$ ). Во втором и третьем столбцах показаны абсолютные результаты для макрокристалла для изохоры $R=1$ и для изобары $P=0$ соответственно. В четвертом и пятом столбцах - относительные (к соответствующим значениям для макрокристалла) значения свойств для кубического нанокристалла из $N=223$ атомов $\left(\right.$ при $\left.N_{p o}=7, f=1\right)$

\begin{tabular}{|c|c|c|c|c|}
\hline Размер $=$ & \multicolumn{2}{|c|}{ Macro $N=\infty$} & \multicolumn{2}{|c|}{ Nano $N=223\left(N_{p o}=7, f=1\right)$} \\
\hline $\begin{array}{c}\text { Свойство } \\
\text { [единица измерения] }\end{array}$ & $\begin{array}{c}R=r_{o} / c=1 \\
P=2.132 \mathrm{GPa}\end{array}$ & $\begin{array}{c}P=0 \\
R=0.9926\end{array}$ & $\begin{array}{c}X(N, R=1) / X(\infty) \\
P(N)=2.118 \mathrm{GPa} \\
P_{s f}=0.014 \mathrm{GPa}\end{array}$ & $\begin{array}{c}X(N, P(N)=0) / X(\infty) \\
R=0.9905 \\
P_{s f}=-0.566 \mathrm{GPa}\end{array}$ \\
\hline $\begin{array}{l}v\left[\mathrm{~cm}^{3} / \mathrm{mol}\right] \\
\Theta[\mathrm{K}] \\
\gamma \\
q \cdot 10^{3} \\
z \\
B_{T}[\mathrm{GPa}] \\
B^{\prime}(P)^{1)} \\
\alpha_{p}\left[10^{-6} \mathrm{~K}^{-1}\right] \\
\alpha_{p} B_{T}\left[10^{-3} \mathrm{GPa} / \mathrm{K}\right] \\
C_{v} /\left(N k_{B}\right) \\
C_{p} /\left(N k_{B}\right) \\
\Theta^{\prime}(P)[\mathrm{K} / \mathrm{GPa}]^{1)} \\
C_{v}^{\prime}(P) /\left(N k_{B}\right)\left[10^{-3} / \mathrm{GPa}\right]^{1)} \\
\alpha_{p}^{\prime}(P)\left[10^{-8} /(\mathrm{K} \cdot \mathrm{GPa})\right]^{1)} \\
C_{p}^{\prime}(P) /\left(N k_{B}\right)\left[10^{-3} / \mathrm{GPa}\right]^{1)} \\
\sigma(100)\left[10^{-3} \mathrm{~J} / \mathrm{m}^{2}\right]^{2)} \\
\sigma^{\prime}(T)_{v}\left[10^{-6} \mathrm{~J} /\left(\mathrm{m}^{2} \mathrm{~K}\right)\right]^{2)} \\
\sigma^{\prime}(T)_{P}\left[10^{-6} \mathrm{~J} /\left(\mathrm{m}^{2} \mathrm{~K}\right)\right]^{2)} \\
\sigma^{\prime}(P)_{T}\left[10^{-3} \mathrm{~J} /\left(\mathrm{m}^{2} \mathrm{GPa}\right)\right]^{122} \\
\sigma /(c \cdot B)^{2)} \\
\mu_{p}^{2)}\end{array}$ & $\begin{array}{r}12.0476 \\
850.185 \\
0.99627 \\
3.74736 \\
1.00371 \\
100.065 \\
4.09232 \\
10.2727 \\
1.02794 \\
2.93007 \\
2.93007 \\
8.46441 \\
-1.95336 \\
-32.4691 \\
-2.91279 \\
1634.05 \\
-45.9160 \\
-72.3402 \\
25.2503 \\
0.16130 \\
0.36934\end{array}$ & $\begin{array}{r}12.3183 \\
831.572 \\
0.99635 \\
3.66472 \\
1.00362 \\
91.2757 \\
4.18437 \\
11.0314 \\
1.00690 \\
2.93654 \\
2.93654 \\
9.06993 \\
-2.02579 \\
-39.2845 \\
-3.18621 \\
1609.61 \\
-45.2743 \\
-74.1784 \\
28.2197 \\
0.17256 \\
0.37789\end{array}$ & $\begin{array}{l}1.0000 \\
0.8836 \\
1.0004 \\
0.8828 \\
0.99957 \\
0.7846 \\
0.9956 \\
1.2845 \\
1.0079 \\
1.0103 \\
1.0103 \\
1.1264 \\
1.0103 \\
1.6202 \\
1.2130 \\
0.9919 \\
1.2868 \\
1.2768 \\
1.2548 \\
1.2547 \\
1.0722\end{array}$ & $\begin{array}{l}1.0064 \\
0.8781 \\
1.0005 \\
0.8772 \\
0.99956 \\
0.7644 \\
1.0018 \\
1.3102 \\
1.0015 \\
1.0108 \\
1.0108 \\
1.1500 \\
1.0216 \\
1.7101 \\
1.2744 \\
0.9876 \\
1.2814 \\
1.2849 \\
1.2938 \\
1.2779 \\
1.0706\end{array}$ \\
\hline
\end{tabular}

Примечание. 1) Рассчитано путем численного дифференцирования данного параметра по давлению вдоль изотермы. 2) Данные параметры рассчитаны при указанных значениях $R$, но при использовании межатомного потенциала пластичного вида, т. е. при $D_{s}$.

Для некоторых свойств макрокристалла кремния при $T=300 \mathrm{~K}$ и $P=0$ из литературы известны следующие экспериментальные оценки

$\Theta /[K]=638-648.8$ [4, стр. 91], 640-678 [19], 645 [20], $963[21]$;

$\gamma=1.0 \pm 0.03[22] ; \gamma_{C}=0.45 \pm 0.05[20]$;

$B_{T} /[\mathrm{GPa}]=97.08[23]-100.75[24]$;

$B^{\prime}(P) 0=4.1[25]-4.72[24]$;

$\alpha_{p} /\left[10^{-6} 1 / \mathrm{K}\right]=7.62[26]-8.76[25]$;

$C_{p} /\left(N k_{B}\right)=2.387[21]-2.415[19]$;

$\sigma(100) /\left[10^{-3} \mathrm{~J} / \mathrm{m}^{2}\right]=1060-2350[4$, стр. 418],

1340-2390 [27];

$\mu_{p}=0.213-0.223$ [28] - для поликристалла;

$\mu_{p}(i, j)=0.180-0.358$ [29], 0.064-0.36 [30] — для различных направлений монокристалла.

Из табл. 2 видно, что наши расчеты хорошо согласуются с данными оценками.

Отметим, что некоторые характеристики, рассчитанные здесь для макрокристалла кремния, оценены впервые, ибо для них до сих пор никаких оценок в литературе нет даже при $T=300 \mathrm{~K}$ и $P=0$. Речь идет об изотермических производных по давлению для функций $\Theta, \alpha_{p}, C_{v}, C_{p}, \sigma(100)$, а также для изохорической и изобарической производных функций $\sigma(100)$ по температуре.

Как видно из табл. 2 и 3, свойства кремния меняются с уменьшением размера по-разному. Слабо изменяются при данных температурах как при изохорическом $R=r_{o} / c=1$, так и при изобарическом $P=0$ уменьшении $N$ следующие параметры: $\gamma, z, B^{\prime}(P)$, $\alpha_{p} B_{T}=(\partial P / \partial T)_{v}, C_{v}, C_{v}^{\prime}(P), C_{p}, \mu_{p}$. Поэтому при различных расчетах можно не учитывать их размерную зависимость, и использовать при расчете свойств нанокристалла кремния значения этих параметров, полученные для макрокристалла. Заметные размерные изменения при $T=300$ и $1000 \mathrm{~K}$ наблюдаются для следующих параметров: $\Theta, q, B_{T}, \alpha_{p}, \alpha_{p}^{\prime}(P), \Theta^{\prime}(P), C_{p}^{\prime}(P), \sigma^{\prime}(T)_{v}$, $\sigma^{\prime}(T)_{P}, \sigma^{\prime}(P)_{T}, \sigma /\left(c B_{T}\right)$.

Исходя из критерия плавления Линдеманна, для относительной температуры плавления можно получить выражение [31]:

$$
T_{m}^{*}=\frac{T_{m}(N)}{T_{m}(\infty)}=\left[\frac{X_{L}(N) \Theta(N) c(N)}{X_{L}(\infty) \Theta(\infty) c(\infty)}\right]^{2}=\left(X_{L}^{*} \Theta^{*} c^{*}\right)^{2}
$$


Полагая, что параметр Линдеманна $\left(X_{L}\right)$ и расстояние между центрами ближайших атомов $(c)$ не зависят от размера нанокристалла, получим $T_{m}^{*}=\left(\Theta^{*}\right)^{2}$. Отсюда и из представленных в табл. 2 и 3 значений $\Theta^{*}$ следует, что величина $T_{m}^{*}$ уменьшается с уменьшением $N$ слабее, чем относительный модуль упругости $B_{T}^{*}$. Подробнее зависимость $T_{m}(N, f)$ для кремния была изучена нами в работе [32].

Отметим, что, как было показано нами на основе $\mathrm{RP}$-модели в $[4,8,11]$, при отклонении формы нанокристалла от наиболее энергетически оптимальной формы (для RР-модели это куб) размерные зависимости свойств нанокристалла усиливаются. Поэтому представленные в табл. 2 и 3 размерные изменения свойств это минимально возможные для указанных термодинамических условий, ибо они рассчитаны для кубической формы нанокристалла со свободной поверхностью Гиббса. Растущие нанокристаллы кремния имеют, как правило, стержневидный либо пластинчатый вид $[32,33]$.

\section{5. Выводы}

Изучено изменение уравнения состояния при изменении размера нанокристалла кремния при $T=300$ и $1000 \mathrm{~K}$. Показано, что при определенном значении относительного объема $\left(v / v_{o}\right)_{0}$ изотермические зависимости $P\left(v / v_{o}\right)$ для нанокристалла и макрокристалла пересекаются.

В точке пересечения (при $\left.\left(v / v_{o}\right)_{0}\right)$ поверхностное давление становится равным нулю: $P_{s f}\left(v / v_{o}\right)_{0}=$ $=P($ Macro $)-P($ Nano $)=0$. Величина $\left(v / v_{o}\right)_{0}$ уменьшается как при изоморфно-изомерном росте температуры, так и при изоморфно-изотермическом уменьшении $N$, или при изомерно-изотермическом отклонении формы нанокристалла от энергетически оптимальной формы (для RP-модели это куб).

Исходя из полученного уравнения состояния, изучено изменение свойств кремния как при изохорическом $\left(R=r_{o} / c=1\right)$, так и при изобарическом $(P=0)$ уменьшении $N$. Показано, что при $T=300$ и $1000 \mathrm{~K}$ параметры: $\gamma, z, B^{\prime}(P), \alpha_{p} B_{T}=(\partial P / \partial T)_{v}, C_{v}, C_{v}^{\prime}(P), C_{p}$, $\mu_{p}$ при переходе от макро- к нанокристаллу изменяются слабо. Поэтому при расчетах можно не учитывать их размерную зависимость и использовать значения этих параметров, полученные для макрокристалла. Заметные размерные изменения при $T=300$ и $1000 \mathrm{~K}$ наблюдаются для следующих параметров: $\Theta, q, B_{T}, \alpha_{p}, \alpha_{p}^{\prime}(P)$, $\Theta^{\prime}(P), C_{p}^{\prime}(P), \sigma^{\prime}(T)_{v}, \sigma^{\prime}(T)_{P}, \sigma^{\prime}(P)_{T}, \sigma /\left(c B_{T}\right), T_{m}$.

Показано, что при изобарическом $(P=0)$ уменьшением размера удельный объем нанокристалла кремния увеличивается тем больше, чем больше температура нанокристалла. Этот вывод согласуется с экспериментальными результатами полученными Гамарником (Gamarnic) [34,35].
Автор выражает благодарность Э.Н. Ахмедову, С.П. Крамынину, Н.Ш. Газановой и 3.М. Сурхаевой за плодотворные дискуссии и помощь в работе.

\section{Список литературы}

[1] Q.S. Mei, K. Lu. Progr. Mater. Sci. 52, 8, 1175 (2007). DOI: 10.1016/j.pmatsci.2007.01.001

[2] L. Liang, M. Li, F. Qin, Y. Wei. Philosoph. Mag. 93, it6, 574 (2013). DOI: $10.1080 / 14786435.2012 .725950$

[3] T. Wang, W. Qi, K. Tang, H. Peng. J. Phys. Chem. Solids. 108, 1 (2017). DOI: 10.1016/j.jpcs.2017.04.010

[4] М.Н. Магомедов. Изучение межатомного взаимодействия, образования вакансий и самодиффузии в кристаллах. Физматлит, М. (2010). 544 с.

[5] М.Н. Магомедов. ФТТ 45, 1, 33 (2003). DOI: $10.1134 / 1.1537405$

[6] Л. Жирифалько. Статистическая физика твердого тела. Мир, М. (1975). 383 c. [L.A. Girifalco. Statistical Physics of Materials. J. Wiley and Sons Ltd, N.Y. (1973).]

[7] М.Н. Магомедов. ФТТ 59, 6, 1065 (2017). DOI: $10.1134 / \mathrm{S} 1063783417060142$

[8] H. Ledbetter, M. Lei, Rao R. Ramji. Physica B: Condens. Matter 159, 3, 265 (1989). DOI: $10.1016 / 0921-4526(89) 90005-7$

[9] М.Н. Магомедов. ЖТФ 80, 9, 150 (2010). DOI: $10.1134 / \mathrm{S} 1063784210090240$

[10] E.N. Akhmedov. J. Phys. Chem. Solids 121, 62 (2018). DOI: 10.1016/j.jpcs.2018.05.011

[11] М.Н. Магомедов. ЖТФ 84, 11, 80 (2014). DOI: $10.1134 / \mathrm{S} 106378421411019 \mathrm{X}$

[12] М.Н. Магомедов. Журн. неорган. химии 49, 12, 2057 (2004).

[13] М.Н. Магомедов. Письма в ЖТФ 39, 9, 9 (2013). DOI: $10.1134 / \mathrm{S} 1063785013050076$

[14] P. Sharma, S. Ganti. J. Mater. Res. 18, 8, 1823 (2003). DOI: $10.1557 / J M R .2003 .0253$

[15] V.M. Huxter, A. Lee, S.S. Lo, G.D. Scholes. Nano Lett. 9, 1, 405 (2008). DOI: 10.1021/n1803275a

[16] C.J. Bhatt, K. Kholiya. Indian J. Pure Appl. Phys. 52, 9, 604 (2014). http://nopr.niscair.res.in/handle/123456789/29354

[17] T. Middelmann, A. Walkov, G. Bartl, R. Schodel. Phys. Rev. B 92, 17, 174113 (2015). DOI: 10.1103/PhysRevB.92.174113

[18] Е.Ф. Пичугин, Изв. вузов. Физика, 6, 77 (1962).

[19] P. Flubacher, A.J. Leadbetter, J.A. Morrison. Philosoph. Mag. 4, 39, 273 (1959). DOI: 10.1080/14786435908233340

[20] W.B. Gauster. Phys. Rev. B 4, 4, 1288 (1971). DOI: $10.1103 /$ physrevb.4.1288

[21] В.М. Глазов, А.С. Пашинкин. Теплофизика высоких температур 39, 3, 443 (2001). DOI: 10.1023/A:1017562709942

[22] А.Ф. Гончаров. Успехи физ. наук 152, 2, 317 (1987).

[23] И.В. Александров, А.Ф. Гончаров, А.Н. Зисман, С.М. Стишов. ЖЭТФ 93, 8, 680 (1987).

[24] Физические величины: Справочник / Под. ред. И.С. Григорьева, Е.3. Мейлихова. Энергоатомиздат, М. (1991). $1232 \mathrm{c}$.

[25] T.C. Pandya, N.A. Thakar, A.D. Bhatt. J. Phys.: Conf. Ser. 377, 012097 (2012). DOI: 10.1088/1742-6596/377/1/012097

[26] С.И. Новикова. Тепловое расширение твердых тел. Наука, М. (1974). 294 c. 
[27] A.A. Stekolnikov, F. Bechstedt. Phys. Rev. B 72, 12, 125326 (2005). DOI: 10.1103/PhysRevB.72.125326

[28] И.Н. Францевич, Ф.Ф. Воронов, С.А. Бакута. Упругие постоянные и модули упругости металлов и неметаллов. Наук. думка, Киев (1982). 286 с.

[29] J.J. Wortman, R.A. Evans. J. Appl. Phys. 36, 1, 153 (1965). DOI: $10.1063 / 1.1713863$

[30] M.A. Hopcroft, W.D. Nix, T.W. Kenny. J. Microelectromech. Systems 19, 2, 229 (2010).

DOI: $10.1109 / J M E M S .2009 .2039697$

[31] М.Н. Магомедов. ЖТФ 78, 8, 93 (2008).

DOI: $10.1134 / \mathrm{S} 1063784208080124$

[32] М.Н. Магомедов. Письма в ЖТФ 42, 14, 94 (2016).

DOI: $10.1134 / \mathrm{S} 1063785016070245$

[33] Е.И. Гиваргизов. Рост нитевидных и пластинчатых кристаллов из пара. Наука, М. (1977). 304 с.

[34] M.Ya. Gamarnic. Phys. Status Solidi B 161, 2, 457 (1990). DOI: $10.1002 /$ pssb.2221610202

[35] M.Ya. Gamarnic. Phys. Rev. B 54, 3, 2150 (1996). DOI: 10.1103/PhysRevB.54.2150

Редактор Т.Н. Василевская 